\title{
A study on Metallo- $\beta$ lactamase producing Imepenem non- susceptible multi-drug resistant Pseudomonas aeruginosa in different clinical specimens in a tertiary care hospital in Kolkata.
}

\author{
Anita Nandi (Mitra),Sujata Bhattacharya,Sampurna Biswas (Pramanik),Saswati \\ Chattopadhyay. \\ 1]Demonstrator, Dept. of Microbiology, Medical College \& Hospital Kolkata \\ 2]Ex-Head of the Dept. Microbiology \&Ex-Professor R.G. Kar Medical College \&Hospital \\ 3] Demonstrator, Dept. of Microbiology, Medical College \& Hospital ,Kolkata \\ 4]Demonstrator, Dept. of Microbiology,IPGME \& R, Kolkata
}

\begin{abstract}
Metallo- $\beta$-lactamase producing carbapenem non-susceptible multidrug resistant Pseudomonas aeruginosa creates a great challenge for treatment as the choice of antibiotics gets severely restricted .This study aimed at detecting such strains in different clinical samples in a tertiary care hospital in Kolkata. Materials and methods-Out of 169 isolates detected as Pseudomonas spp.in Microbiology laboratory 98 consecutive non-duplicate isolates phenotypically confirmed as Pseudomonas aeruginosa were selected following inclusion criteria of being resistant to $\geq 3$ different classes of antibiotics including Imepenem. Antibiogram with antibiotics suggested by CLSI-2011 was performed and resistance to different antibiotics was recorded .Then combined disc test with Imepenem $(10 \mu \mathrm{g})$ and Imepenem $(10 \mu \mathrm{g})+E D T A(750 \mu \mathrm{g})$ was performed.Observation - Strains showing zone difference $\geq 7 \mathrm{~mm}$ during combined disc test were considered as metallo- $\beta$ lactamase producing multidrug resistant strains and those showing $<7 \mathrm{~mm}$ increase in zone size or no increase in zone size or total zone size less than $13 \mathrm{~mm}$ with Imepenem+EDTA were considered as MBL nonproducers.51 isolates out of 98(52.04\%) multidrug resistant oneswere diagnosed as MBL producing multidrug resistant isolates. Sample wise distribution showed highest rate of infection with imepenem non-susceptible multidrug resistant Pseudomonas aeruginosa was seen in fluid samples(66.66\%) and lowest rate (14.28\%) in blood sample isolates.Conclusion-The finding of 52.04\% MBLproducing isolates out of 98 multidrug resistant Pseudomonas aeruginosa is an alarming condition in the background of tertiary care hospital and may be the cause of high mortality and morbidity rate.

Key words: Pseudomonas aeruginosa,metallo- $\beta$ lactamase(MBLs), multidrug resistant(MDR), phenotypic characterisation.
\end{abstract}

\section{Introduction:}

Pseudomonas aeruginosa is a virulent agent having a tendency to develop resistance to majority of the antibiotics available for treatment. It is a leading cause of life- threatening nosocomial infection [1]. Its intrinsic resistance to many antimicrobial agents and development of multidrug resistance impose severe therapeutic problem for clinicians[2] .Presently Carbapenems are very useful antimicrobial agents for treatment of the multidrug resistant Pseudomonal infection; however increasing \& irrational uses of these agents resulted in the development of carbapenemresistancebyPseudomonas aeruginosa[1].Carbapenems have high affinity for PBP 2 , stability to most $\beta$-lactamases and excellent permeation across bacterial outer membranes [3]. Production of intrinsic Carbapenem hydrolyzing enzymes by Pseudomonas aeruginosa is an important cause of its drug resistance. Molecular studies revealed that the carbapenem hydrolyzing enzymes belong to Ambler group A, B, C \& D. Group B produces Metallo- $\beta$-lactamase (MBLs), an enzyme requiring divalent cation Zinc as co-factor and is inhibited by action of metal ion chelators. The MBLs hydrolyse betalactams except Aztreonam in vitro[5]. The first MBL was detected in Japan in early 1990s [1,4].Originally it was thought to be uncommon and restricted to some specific geographical areas but presently acquired MBLs are known to be widespread .The presence of these enzymes have been reported from various parts of the world including Asia,Europe, Australia,South and North America [5].As carbapenems are now frequently used in treatment of multidrug resistant bacterial infections in hospital environment, the hospital strains of Pseudomonasspp.are more exposed to these group of antibiotics and the greater is the chance of development of resistance. Our study aimed at search for metallo- $\beta$ lactamase producing multidrug resistant Pseudomonas aeruginosa showing resistance to more than three different classes of antibiotics including Imepenem,(a frequently used carbapenem in our healthcare set up) in different clinical samples sent to Microbiology laboratory.High antibiotic pressure induces development of drug resistance and spread of that resistance by horizontal gene transfer leads to quick development of resistance to multiple antibiotics in hospital environment 
[6]. Pseudomonas aeruginosa often causes opportunistic infection in immunocompromised patients. Surgical wound infection, burn wound infection, neonatal septicaemia ,pneumonia in patients with cystic fibrosis and inpatients with ventilator support; septicaemia in patients with central venous catheter or ventriculo-peritoneal shunt, urinary tract infection in catheterised patients are often associated with P.aeruginosa. Multidrug resistant strains especially MBL producing ones are really a great threat to them because very few treatment options are left . The prevalence of colonisation by Pseudomonas aeruginosa in healthy subjects is usually low, but higher colonisation rate can be encountered following hospitalisation especially among subjects treated with broad spectrum antibiotics and in immunocompromised patients[7].our specimens were selected from different types of lesions. Early detection of MBL producing organisms is of crucial importance for prevention of inter- and intra-hospital disseminations not only in institutions with high prevalence of such isolates but also in those in whichphenotype of resistance have never been detected .There are several phenotypic process of identifying MBLs [4]-- (a) Double disc synergy test (b) Combined disc test,(c) Modified Hodge test(d)MBL E-test\& (e) Microdilutionaltest.We selected combined disc test method.

\section{Materials \&Methods.:}

Specimens from patients of different departments like Medicine, Surgery, Gynaecology \& Obstetrics , Orthopaedics, Paediatrics, Casualty, intensive care unit, post-anaesthetic care unit, burn unit and ENT ward sent to our department were taken for study. Non-duplicate isolates of Pseudomonas spp.diagnosed during routine laboratory work showing resistance to 3 or more different antibiotic classes including Imepenem were selected from(1)superficial wound, surgical wound, burn wound(2) urine (3) blood of septicaemia cases (4) sputum(5) deep tracheal aspirate, broncho-alveolar lavage (6) Tip of Foley's catheters, sucker tubes \&V-P shunt (7) pus of ear infection. Strains of Pseudomonas spp. resistant to $\geq 3$ antibiotic classes including Imepenem were collected for aperiod of one year from July 2011 to June 2012 .The study was longitudinal ,prospective and randomized type. The study protocol complied with the Declaraton of Helsinki and was approved by Institution's ethical committee.

\section{Identification of strains :}

Ninety eight strains were collected from 169 isolates of Pseudomonas spp . Isolates identified as Pseudomonas spp.were further confirmed as Pseudomonas aeruginosa by standard laboratory tests--- Gram stain, motility test, pigment production in nutrient agar, growth at $42^{0} \mathrm{c}$, growth in selective media of Cetrimide agar with $0.5 \%$ Nalidixic acid (Himedia Ref M04-100G), specific odour from colonies, oxidase test, catalase test, oxidation-fermentation reaction test, nitrate reduction test and arginine dehydrolation test

\section{Antibiogram}

All strains selected for the study were tested with six classes of antibiotics according CLSI 2011 guidelines -Imepenem( $10 \mu \mathrm{g})$, Ceftazidime $(30 \mu \mathrm{g})$,Piperacillin $(100 \mu \mathrm{g})$, Tobramycin $(10 \mu \mathrm{g})$, Gentamicin $(10 \mu \mathrm{g})$ \& Cipro-floxacin $(5 \mu \mathrm{g})$.Antimicrobial susceptibility was detected by Kirby- Bauer disk diffusion method. Inhibition zones were interpreted according to CLSI guidelines. ATCC Pseudomonasaeruginosa(27853) was used as control of antibiotic sensitivity test.

\section{Combined disc test}

There is no specific guide line of CLSI for detection of metallo- $\beta$ - lactamases. So the procedure was followed as per method described by Yong D et al2002 [8] and J. Pitout 2005[4] A bacterial suspension of test strain in peptone water was incubated at $37^{\circ} \mathrm{C}$ for $2 \mathrm{hrs}$; turbidity was matched with $0.5 \mathrm{McFarland}$ turbidity standard and then inoculated in Muller-Hinton agar. Imepenem $(10 \mu \mathrm{g})$ and Imepenem-EDTA $(10 \mu \mathrm{g}+750 \mu \mathrm{g})$ discs were placed at $15 \mathrm{~mm}$ distance from edge to edge to detect MBL production by difference of $\geq 7 \mathrm{~mm}$ between zone of inhibition by Imepenem and Imepenem- EDTA. Accordingly the result was tabulated. Imepenem non-susceptible strains showing $\geq 7 \mathrm{~mm}$ increase in zone of inhibition with Imepenem-EDTA were considered as MBL producers. Those isolates showing zone of inhibition $<7 \mathrm{~mm}$ or no zone of inhibition or total zone of inhibition $<13 \mathrm{~mm}$ with Imepenem-EDTA disc were MBL nonproducers. ATCC P.aeruginosa (27853) was taken as negative control and lacal strains of Klebsiella spp. as positive control for detection of MBL activity.

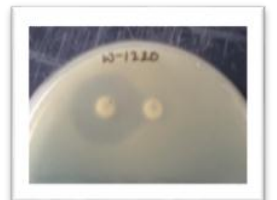

Disc of Imepenem \&Imepenem+EDTA; increased zone of inhibition seen around Imepenem-EDTA disc 


\section{Results:}

Total niney-eight (98) non-duplicate consecutive strains of Pseudomonas aeruginosa showing resistance to $\geq 3$ antibiotic classes including Imepenem were selected for study from 169 isolates of Pseudomonas spp detected during the period of one year from July 2011 to June 2012. These 98Imepenem non-susceptible multidrug resistant isolates were identified in following specimens.(Table 1)

Table 1:Specimen distribution and percentage

\begin{tabular}{|c|c|c|c|c|c|c|}
\hline Specimen & pus & sputum & urine & blood & fluids & others \\
\hline number & 58 & 14 & 13 & 3 & 7 & 3 \\
\hline percentage & $59.08 \%$ & $14.28 \%$ & $13.26 \%$ & $3.06 \%$ & $7.77 \%$ & $3.03 \%$ \\
\hline
\end{tabular}

The 58 pus specimen came from Male and Female surgical wards, Casualty ward, Gynaecology \&Obstetrics ward , Cardio-thoracic and vascular surgery ward(CTVS), Orthopaedic surgery ward ,uro-sugery ward and ENT ward . No specimen was obtained from Eye ward. Sputum specimens were obtained from male and female Medicine ward,Chest ward and CTVS. Urine specimens were from medicine (male\& female) \& surgery (male female) wards and from uro-surgery ward where these were collected from catheterized patients. Blood specimens were sent from male surgical, female medicine ward and ICU. Among 'Fluid specimens" deep-tracheal aspirates \& broncho-alveolar lavage fluid came mainly from ICU, PACU and CTVS ward. 98 isolates collected from Microbiology laboratory were confirmed as Pseudomonas aeruginosa phenotypically. These were subjected to antibiotics prescribed by CLSI guidelines 2011 for P.aeruginosa.

Table 2: Resistance pattern to different classes of antibiotics

\begin{tabular}{|l|c|l|l|}
\hline Antibiotics & $\begin{array}{l}\text { Resistant } \\
\text { isolates }\end{array}$ & $\begin{array}{l}\text { Susceptibles } \\
\text { isolates }\end{array}$ & $\begin{array}{l}\text { \% of } \\
\text { resistant isolates }\end{array}$ \\
\hline Imepenem & 98 & nil & 100 \\
\hline Ceftazidime & 82 & 16 & 83.67 \\
\hline Piperacillin & 63 & 35 & 64.28 \\
\hline Tobramycin & 60 & 38 & 61.22 \\
\hline Gentamicin & 82 & 16 & 83.67 \\
\hline Ciprofloxacin & 75 & 13 & 76.53 \\
\hline
\end{tabular}

Antibiotic sensitivity test result(Table 2) showed all isolates were resistant to Imepenem and also exhibited resistance to three, four ,five or six different classes of antibiotics. Resistance to Ceftazidime was $83.67 \%$, to Piperacillin $64.28 \%$, to Tobramycin $61.22 \%$, to Gentamicin $83.67 \%$ and to Ciprofloxacin $76.53 \%$. These isolates were then subjected to Combined Disc Test withImepenem $(10 \mu \mathrm{g})$ and Imepenem + EDTA(750 $\mu \mathrm{g}) .51$ isolates were Phenotypically identified as Metallo- $\beta$-Lactamase type Carbapenemase producing strains as they showed $\geq 7 \mathrm{~mm}$ increase in zone of inhibition with Imepenem-EDTA disc.47 isolates showing $<7 \mathrm{~mm}$ zone difference or no zone of inhibition or zone of inhibition with Imepenem-EDTA less than $13 \mathrm{~mm}$ were phenotypically identified as MBL non-producer type Imepenem resistant strains(Table $3 \& 4$ )

Table 3 :Combined disc test result

\begin{tabular}{|l|c|c|}
\hline Isolates & MBL producers & MBL non- producers \\
\hline Numbers & 51 & 47 \\
\hline Percentage & 52.04 & 47.96 \\
\hline
\end{tabular}

Table 4 :Specimenwise distribution of MBL producing isolate

\begin{tabular}{|l|c|c|c|c|c|c|}
\hline Specimen & pus & sputum & blood & urine & fluids & others \\
\hline Total no & 58 & 14 & 7 & 13 & 3 & 3 \\
\hline MBLproducers & 32 & 7 & 1 & 8 & 2 & nil \\
\hline percentage & 55.17 & 50 & 14.28 & 61.54 & 66.66 & 0 \\
\hline
\end{tabular}

\section{Discussions}

Drug resistance of infective agents, specially multidrug resistance is a matter of serious concern as it results in increased morbidity and mortality of the patients resulting from treatment failure and also increases health-care cost and loss of man-hour in productivity. Increased morbidity of the patients lengthens the bed-disability days, decreases the bed-turnover rate in hospitals and also increases cost of therapy of such difficult to treat infections. In immunocompromised patients or very young and old aged patients infection with multidrug resistant strains of any bacteria may even cause septicaemic shock, multi organ failure leading ultimately to fatal outcome. Wide spread usage of antibiotics exerts a selective pressure that acts as a driving force in the development of antibiotic resistance. In hospital environment injudicious use of 
antibiotics empirically or after proper culture and sensitivity test is much more than that in the community. So the nosocomial infections are faced with multidrug resistance very frequently. Resistance factors, particularly those carried on mobile genetic elements can rapidly spreads to human and animal populations. These types of pathogens are present not only locally, but they spread globally also. Among several bacteria developing multidrug resistance Pseudomonas aeruginosa is very much vulnerable for its intrinsic nature of resistance to different classes of antibiotics including carbapenems which is one of theanti-pseudomonal drug as well as drug of choice for multidrug resistant strains of bacteria. Sometallo- $\beta$ lactamase producing Carbapenem non-susceptible multidrug resistant strains of Pseudomonas aeruginosa are considered as highly virulent. In present study over a period of one year from July 2011 to June 2012 from 169 isolates of Pseudomonas spp. 98 non-duplicate multidrug resistant Imepenem non-susceptible isolates were selected as per inclusion criteria of being resistant to $\geq 3$ different classes of antibiotics including Imepenem. These were detected in different clinical specimens sent to the Microbiology department from different departments. Among ninety-eight selected isolates fifty-eight isolates were detected from pus specimens of surgical wounds, abscesses or discharge from ear infection. It constituted $59.18 \%$ of total Imepenem nonsusceptible MDR strains. These were collected from patients of Male \& Female surgical ward, casualty ward, orthopaedic surgery ward, Gynaecology \& Obstetric ward, cardio -thoracic vascular surgery(CTVS) ward and ENT ward. Fourteen sputum specimens (14.28\% ) came from male \& female medicine ward, chest ward and CTVS ward. Thirteen urine specimens constituting $13.26 \%$ of selected isolates were from female surgical ward and medicine ward and from uro-surgery ward where urine was collected from catheterised patients with proper precaution.Three isolates constituting $3.06 \%$ of total Imepenem nonsusceptible MDR strains were from blood of septicaemic patients admitted in male surgical, female medicine and intensive care unit. Seven strains isolated from deep tracheal aspirates and broncho-alveolar lavage fluid of patients in ICU, PACU and CTVS ward contributed 7.7\% of MDR strains detected by Kirby-Bauer disc diffusion technique. Tip of ventriculo-peritoneal shunt, sucker tube and Foley's catheter sent for culture \& sensitivity have been mentioned as 'Other' samples. All isolates were resistant to Imepenem. Resistance seen to Ceftazidime was $83.67 \%$,toPipercillin was $64.28 \%$, to Tobramycin was $61.22 \%$, to Gentamicin was $83.67 \%$ and to Ciprofloxacin was $76.53 \%$ of isolates. All of these isolates showing resistance to 3 or more than 3 different classes of antibiotic including Imepenem (carbapenem) were selected for study to identify metallo- $\beta$-lactamase activity in them by phenotypic method. The isolates were subjected to battery of phenotypic tests to establish them as Pseudomonas aeruginosa. No other species of genus Pseudomonas was identified during the tests. Then six different classes of antibiotics were applied to them for sensitivity test by Kirby -Bauer disc diffusion method as per CLSI guide-lines 2011.Imepenem , Ceftazidime ,Piperacillin ,Tobramycin, Gentamicin and Ciprofloxacin were selected for test. Resistance to $3,4,5$ and even all 6 classes were observed. So phenotypically all 98 isolates were confirmed as Imepenem non-susceptible multidrug resistant P.aeruginosa. To identify metallo- $\beta$ - lactamase activity in these isolates Combined disc test using Imepenem $(10 \mu \mathrm{g})$ and Imepenem-EDTA $(10 \mu \mathrm{G}+750 \mu \mathrm{g})$ was chosen (Yong et al2002) [8] .This Imepenem-EDTA disc is available in the market. It does not require preparation, sterilisation or storage of EDTA and then application to blank filter paper disc for preparation of EDTA disc as required for double disc synergy test which is thus a time-consuming test (Yong 2002) [8]. Combined disc test (CDT) is simple ,rapid, easy and economic process for laboratory work to identify MBL activity at the earliest incidence[9]. Previous work of Ting- ting Qu et al (2009) [10] and L.Berges et al 2007[11] showed that $292 \mu \mathrm{g}$ EDTA per disc gave only 3-4 mm increase in zone of inhibition during comparative study where as750 $\mu \mathrm{g}$ and $930 \mu \mathrm{g}$ EDTA increased the zone-inhibition by more than $7 \mathrm{~mm}$. EDTA itself has an antibacterial activity. So higher dose of $930 \mu$ gwas not choosen .The comparative study of 98 samples showed two groups--MBL producers \&MBL non-producers. The fifty one isolates showing $\geq 7 \mathrm{~mm}$ zone difference between Imepenem and Imepenem- EDTA disc were diagnosed phenotypically as metallo- $\beta$-lactamase producing strains. The 47 isolates showing $<7 \mathrm{~mm}$ zone difference, no zone difference or zone of inhibition with Imepenem-EDTA $\leq 13 \mathrm{~mm}$ were considered as non-MBL producing Imepenem resistant strains. 51 phenotypic MBL producing strains constituted 52.04\% of total Imepenem non-susceptible MDR isolates included in study and 47 MBL non-producer type isolates constituted $47.96 \%$. These MBL non-producers developed resistance to Imepenem by some means other than MBL type cabapenemase production.The phenotypic studies in India have shown incidence of MBL producing Pseudomonas aeruginosa causing wound infection, septicaemia, urinary tract infection, pneumonia, neonatal septicaemia vary from $6 \%-69 \%$ $[12,13,14,15,16, \& 17]$. The present study also showed finding similar to them. Specimen wise distribution showed $55.17 \%$ of pus $50 \%$ of sputum, $14.28 \%$ of blood, $61.54 \%$ of urine and $66.66 \%$ of fluid specimens harboured the metallo- $\beta$ lactamase producing Imepenem non-susceptible multidrug resistant Pseudomonas aeruginosa. K.Prabhat Rajanet al(2010)[13] found prevalence rate of 32.04\% Pseudomonas aeruginosa infection in case of surgical and burn wound . In the present study the $55.17 \%$ of pus sample showed presence of MBL producing multidrug resistant Pseudomonas aeruginosa. 50\% of the sputum samples of the patients of 
male and female medicine ward, cardiothoracic vascular surgery ward and chest ward harboured MBL producing P.aeruginosa.61.54\% of urine specimens of male \& female surgical ward and uro-surgery ward showed evidence of similar isolates. Highest rate of infection(66.66\% )with MBL producing Imepenem nonsusceptible multidrug resistant P.aeruginosa infection was seen in fluid samples which were mainly deep tracheal aspirates and broncho-alveolar lavage fluid collected from patients of intensive care unit, post anaesthetic care unit, cardio thoracic vascular surgery unit whereas lowest incidence was seen in blood samples $(14.28 \%)$.This is a grave situation for tertiary care providing health care setup.

\section{Conclusion}

Tertiary care providing health care set up deals with different types of patients many of whom are predisposed with morbidity or mortality increasing factors . Infection with metallo- $\beta$ lactamase producing multi drug resistant Pseudomonas aeruginosa in such conditions leave almost no or very little treatment option. The development of such drug resistance could be controlled by taking highest level of care in selecting antimicrobial agents and following strict antibiotic policy.

\section{Acknowledgement}

I express my gratitude to ex-Professor\& ex-Head of the dept. Microbiology Dr.Sujata Bhattacharya who was my guide in performing this research work as dissertation during my post graduate trainee period. I have been supported by the Microbiology laboratory of R.G. Kar Medical College \& Hospital where I completed my M.D. in Microbiology. The expenses of the research work was borne partly by government and partly by myself.

\section{References:}

[1]. Mariana Castenhier,Mark A Toleman ,Ronald N.Jones et al. Molecular Characterization of a Beta-lactamase gene,bla ${ }_{\text {GiM-1 }}$ Encoding a new subclass of MBLs. Antimicrobial agents and chemotherapy Dec 2004 ;48(12):pg4654-4661.

[2]. Gales,A.C , R.N.Jones , J.Turnidge et al.Characterisation of Pseudomonas aeruginosa isolates : occurrence rate, antimicrobial susceptibility pattern and molecular typing in the global SENTRY. Antimicrobial surveillance programme 1997-1999:Clinical infectious Disease:32 (suppl:2)S146- S155.

[3]. Yochi Hirakata, Koichi Izumikawa et.al . Rapid detection and evaluation of clinical characteristics of Emerging Multidrug resistant Gram Negative Rods carrying metallo-beta-lactamaseGeneblaIMP.Antimicrobial agents and chemotherapy Aug 1998 ; (8) : pg 2006-2011.

[4]. Johann D.D.Pitout, Daniel B.Gregson,LaurentPoirel et al. Detection of Pseudomonas aeruginosa Producing Metallo-BetaLactamases in a large centralised Laboratory . Journal of Clinical Microbiology July 2005;43:(7):pg 3129-35 .

[5]. HorishSederil,ZohrehKarmil et.al. Phenotypic detection of Metallo- $\beta$-lactmases producing Pseudomonas aeruginosa strains isolated from burned patients. Iranian Journal of Pathology.2008 ; $3:$ (1) :822-25

[6]. Fred C. Tenover. Mechanisms of Antimicrobial Resistance in Bacteria.The American Journal of Medicine (2006); Vol 119 :6A: S3-S10

[7]. G. M. Rossolini and E. Mantengoli .Treatment and control of severe infections caused by multiresistantPseudomonasaeruginosa . J Clinical Mirobiology and Infection.2005:vol 11:(supplement 4):pg 132-138.

[8]. Yong D, Lee K, Yum JH, Shin HB , Rossolini GM , ChogY.Imepenem- EDTA disc method for differentiation of Metallo- $\beta$ lactamase producing clinical isolates of Pseudomonas aeruginosa and Acinetobactor spp. J. Clinical Microbiology .2002;40: (10) : pg 3798-3801.

[9]. Singh SP, Shariff M, Barua T, ThukralSS.Comparative evaluation of phenotypic tests for identification of metallo $\beta$-lactamases producing clinical isolates of Pseudomonas aeruginosa.Indian J Med Res 2009;129:713-15.

[10]. Ting-ting Qu, Jun-li Jie Wang, Jing Tao, et al .Evaluation of Phenotypic Tests for Detection of Metallo- $\beta$-Lactamase-Producing Pseudomonas aeruginosa Strains in China. J. Clin. Microbiol. April 2009 47: (4) :1136-1142.

[11]. L. Bergès, H. Rodriguez-Villalobos, A. Deplano, and M. J. Struelens. Prospective evaluation of imipenem/EDTA combined disc and Etest for detection of metallo- $\beta$-lactamase-producing Pseudomonas aeruginosa.J. AntimicrobChemother April 2007; 59: (3):812-813.

[12]. Simit H. Kumar, Anuradha S. De ,SujataBaveja and Madhuri A. Gore . Prevalence and risk factors of Metallo- $\beta$-Lactamase producing Pseudomonas aeruginosa and Acinetobactor species in Burns and Surgical wards in a tertiary care hospital. J. Lab Physicians 2012;Jan-June :4 : (1):39-42.

[13]. K.prabhatRanjan ,NeelimaRanjan ,Satish K Bansal and D.R. Arora .Prevalence of Pseudomonas aeruginosa in Post-operative Wound infection in referral hospital in Haryana,India. J. Lab. Physicians Jul-Dec 2010; 2 : (2):74-75.

[14]. DurgeshGopalRao, Deshmukh, AjitS.Damle et al. Metallo- $\beta$-lactamase producing clinical isolates from Patients of a Tertiary care hospital.J Lab Physicians. 2011 Jul-Dec; 3(2): 93-97.

[15]. Madhu Sharma, SaritaYadav and Uma Chowdhury .Metallo- $\beta$ lactamase producing Pseudomonsaeruginosa in Neonatal Septicaemia. J . Lab .Physicians 2010; 2(1):14-16.

[16]. Rahul Mittal, SudhirAggarwal, SarojSharma . Urinary tract infections caused by Pseudomonas aeruginosa :a mini review Journal of Infection and Public Health2009;2:101-111.

[17]. Sakshi P Singh ,MaliniShariff ,TanushreeBarua \& \& S.S. Thukral. Comparative evaluation of phenotypic tests for identification of Metallo - $\beta$-Lactamase producing clinical isolates. Indian J. Medical Research:June 2009; 129: (6) : pg 713-15 . 